\title{
Eight unanswered questions about the COVID-19 pandemic
}

\author{
We asked experts around the world what surprised them about SARS-CoV-2 and COVID-19, and what questions \\ remain unanswered.
}

\section{Linfa Wang: Bat immunology}

Linfa Wang is a professor in the Emerging Infectious Diseases Program, DukeNational University of Singapore Medical School, Singapore.

From day one, when this mysterious pneumonia started happening in late 2019, I thought the causative agent could be another coronavirus - most likely another bat coronavirus. By the end of 2019, I had been studying bat coronaviruses for almost 17 years, and it's no surprise anymore for people like me to see another animal coronavirus jumping into humans. From serological data from humans, I know that spillover of viruses from bats to humans happens often, but most of that spillover happens in remote areas, and it usually either is not very transmissible or doesn't cause severe disease. But if any of these viruses get to a major city with lots of wildlife trading, the virus can adapt, via an animal intermediate host, allowing human-to-human transmission.

When spillover causes an outbreak of human infections, there are three types of viruses to consider: ancestral, progenitor, and outbreak. We know the ancestral virus in bats and the outbreak virus in humans (SARS-CoV-2), but not the progenitor virus - the virus that allowed the spillover. So, we need to focus on identifying the early transmission event.

Our lab developed a serological test for neutralizing antibodies to SARS-CoV-2. This can find antibodies that defend against the virus. With a large collection of pre-pandemic samples, serology could be used for origin studies. If we could randomly take a large number - for example, 10,000 - of pre-pandemic samples from different parts around the world, we could see when and where spillover occurred and determine which samples are the closest to the outbreak virus, SARS-CoV-2. This serosurveillance can be done only if the international community is willing to provide samples, but it's going to be very difficult, if not impossible. Nobody wants to say COVID-19 started in their country.

Although the world is focused on bats as the source of this pandemic, I'd like to emphasize that we could learn so much

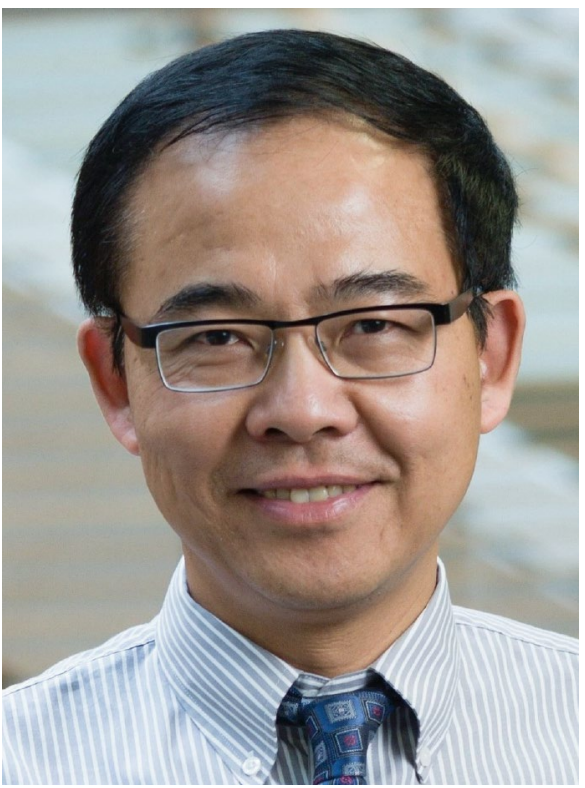

Linfa Wang. Credit: Duke-NUS Communication Group

from these animals. Bats are well known for studies of flight and echolocation, but they are also important reservoirs of viruses, although they are not susceptible to diseases caused by most viruses - with rabies as the main exception. Bats could be used as a model system for studying immunological defense in mammals, and these studies would surely benefit human health.

\section{Bryan Grenfell, Caroline Wagner and Chadi Saad-Roy: New variants}

Bryan Grenfell is the Kathryn Briger and Sarah Fenton Professor of Ecology and Evolutionary Biology and Public Affairs at the School of Public and International Affairs, Princeton University; Caroline Wagner is an assistant professor in the Department of Bioengineering, McGill University; Chadi Saad-Roy is a doctoral candidate at the Lewis-Sigler Institute for Integrative Genomics, Princeton University.

The population dynamics of SARS-CoV-2 are strongly influenced by a variety of parameters, including characteristics of immunity after natural infection or vaccination, availability of vaccines, non-pharmaceutical interventions, potential seasonal climatic or behavioral drivers, and - perhaps most importantly emerging viral variants. In all likelihood, the virus will remain in society for a long time, and population dynamics may eventually equilibrate to some sort of endemic seasonal and/or longer-term epidemic cycle, given experience with previous acute pandemic and endemic diseases. However, we don't know how long this shift from pandemic to endemic or epidemic will take, nor what the characteristics of the steady state will be - or how vaccination strategies will affect these patterns.

One surprise was how much non-pharmaceutical interventions for COVID-19 reduced the transmission

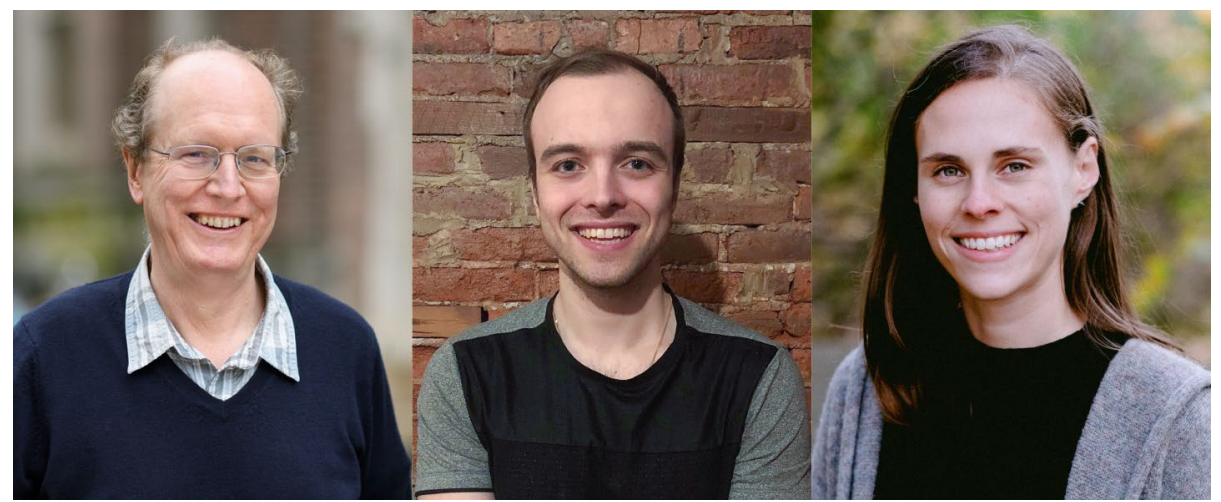

Bryan Grenfell (left), Chadi Saad-Roy (middle) and Caroline Wagner (right). Credit: Sameer Khan (left), Kristin Hauge (middle) and Marlon Kuhnreich photography (right) 
of other respiratory infections, such as infection with influenza virus and respiratory syncytial virus, viruses that are now bouncing back.

Real-world studies have shown the considerable impact of vaccines, but the way vaccines are distributed strongly influences global population dynamics. Large pockets of unvaccinated individuals, particularly when they are spatially clustered, can drive large outbreaks. Our models, and a variety of other studies, illustrate that vaccine equity is crucial. Allowing transmission in unvaccinated populations presents an ideal scenario for pathogen evolution and new variants of concern, which ultimately threatens gains in immunity around the world, including in highly vaccinated populations.

There are several key unanswered questions about the evolution of SARS-CoV-2. In which populations are new variants occurring? What are the characteristics of breakthrough infections? In particular, what's the susceptibility of vaccinees relative to that of unvaccinated people, and are breakthrough infections less transmissible? Are infections more likely to occur for different immune profiles, depending on prior natural infection, specific vaccination and specific viral strains? How long do natural and vaccinal immunity last - in terms of both clinical protection and infection?

We still don't fully understand the dynamics and impact of new variants, but we are beginning to incorporate increasing amounts of real-world data related to immune parameters into our epidemic models, as well as continuing to refine how we incorporate human behavior.

\section{Kathryn Edwards: Better data on boosters}

Kathryn Edwards is a professor of pediatrics, the Sarah H. Sell and Cornelius Vanderbilt Chair, and the scientific director of the Vanderbilt Vaccine Research Program, Vanderbilt University Medical Center.

The really hard question is how we are going to live in the future with this pathogen, as SARS-CoV-2 is likely to be a permanent endemic virus. In the short term, everyone needs to get vaccinated. It is clear that areas with lower vaccination rates are having higher burdens of disease and higher death rates. What we don't know is whether everybody needs to be boosted or how often everybody needs to be boosted. Understanding the need, or not, for boosters, will require that the research community and policymakers have an open mind, and let the data tell us what we need to do.

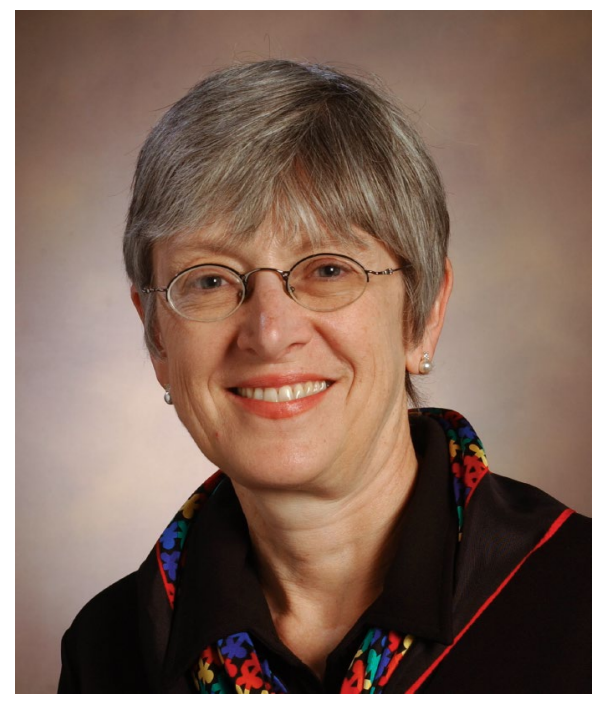

Kathryn Edwards. Credit: Kathryn Edwards

Worries from the public about vaccine safety are an ongoing concern. All the vaccine companies and the Centers for Disease Control and Prevention (CDC) are calling on people who have been studying vaccines all their life to make sure of safety, which has included my working on a CDC contract to look at vaccine safety, and on the safety monitoring committee for Pfizer. It is important that researchers continue to better understand when adverse events happen, how could they be prevented and what the reasons for the adverse events are.

But overall, vaccines for COVID-19 are incredibly, incredibly safe. There have been some safety signals, but they've been quite rare - just a few in a million. Those of us who work on vaccine safety need to better communicate how seriously we take assessing safety and making sure that the vaccines are as safe as they can be. If the public understands and appreciates this work, then this can reduce vaccine hesitancy.

Manoj Murhekar: Reasons for hesitancy Manoj Murhekar is the director of the National Institute of Epidemiology, Indian Council of Medical Research, Chennai.

One unexpected challenge early in the pandemic was the stigma associated with COVID-19. Some people were reluctant to get tested, fearing isolation or quarantine. This even affected healthcare workers, who faced hardships and alienation because of their vulnerability to catch SARS-CoV-2.

India has a huge population, with about 940 million adults eligible to receive a vaccine against COVID-19. As of September 2021, nearly $70 \%$ of the eligible individuals had received one dose and $25 \%$ had received both doses. Sustaining the

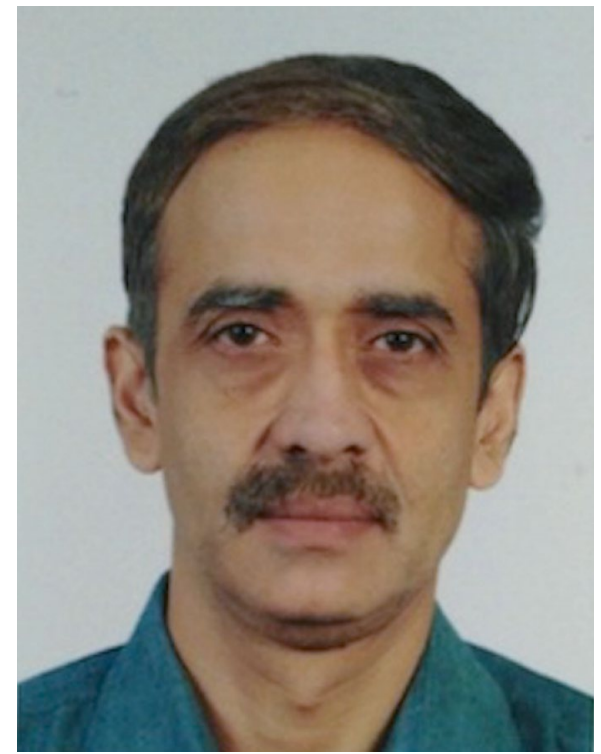

Manoj Murhekar. Credit: Manoj Murhekar

pace of vaccination to cover the remaining unvaccinated population is the greatest challenge facing India in 2021 and 2022.

Despite the availability of effective and safe vaccines, and even after seeing their near and dear ones dying, some sections of society are hesitant to receive a vaccine. More research is needed to understand the reasons for vaccine hesitancy and how to tackle it.

\section{Malik Peiris: Mucosal immunity}

Malik Peiris is a professor of virology at the School of Public Health, and a co-director of the World Health Organization H5 Reference Laboratory and SARS-CoV-2 Reference Laboratory, University of Hong Kong.

Although we have learned a lot about transmission of SARS-CoV-2, questions still remain. We still don't know the relative contributions of small airborne aerosols versus droplets versus fomites in transmission. Clearly, the contribution of different modalities differs depending on context and the environmental setting. We also do not know how the more infectious variants, such as Delta, increase transmission. It could arise from an increased viral load shed from infected persons, increased duration of infectiousness or increased survival of infectious virus in the aerosols.

We also need to know more about the impact of various public-health measures, such as how much different types of masks affect transmission. Similarly, we don't yet fully understand how much different vaccines reduce transmission and what is 


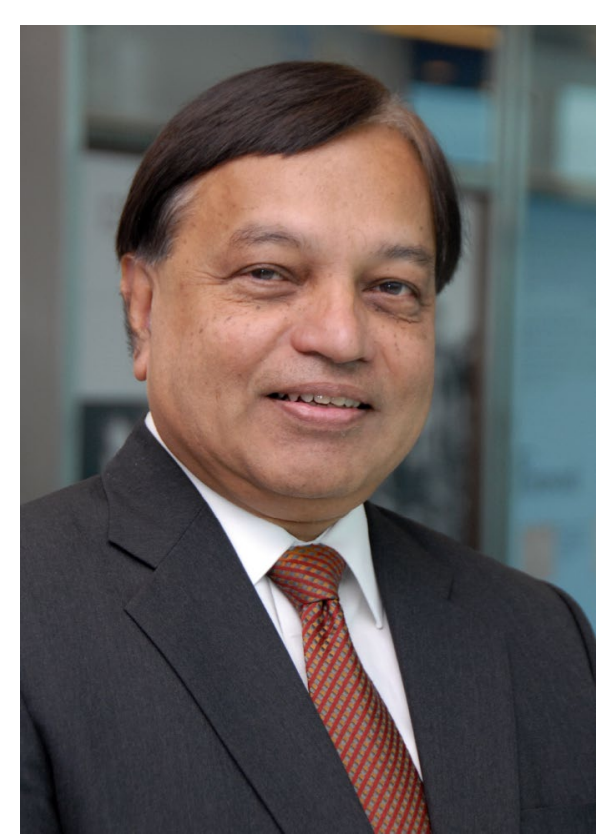

Malik Peiris. Credit: Malik Peiris

the durability of this impact, and if it differs with the vaccine type and viral variants.

It is clear that currently available vaccines alone do not entirely prevent transmission. But what happens when vaccinated individuals get - hopefully mild - breakthrough infections? Vaccine immunity followed by natural infection even if asymptomatic - may elicit enhanced transmission-blocking immunity because the mucosal immune system is now engaged after infection via the nose and throat. If vaccination followed by a usually mild breakthrough infection provides broadened immunity to or protection against a range of variants, we may be seeing light at the end of the tunnel.

This raises questions about the best route of delivery of COVID-19 vaccines. Injected vaccines do provide good immunity against severe disease, but that is not the ideal route for eliciting immunity in the nasopharyngeal mucosa, which is needed for full protection from infection and transmission. An intranasal vaccine could be more successful in preventing transmission. Perhaps a combination of conventional injected vaccines and intranasal vaccines would elicit both systemic immunity and mucosal immunity that will provide better protection from infection and reduce transmission.

\section{Ester Cerdeira Sabino: Political obstacles}

Ester Sabino is a professor at the Institute of Tropical Medicine, University of São Paulo, Brazil.
In Brazil, the autochthonous transmission of the Delta variant started in Rio de Janeiro in June 2021 and then spread across the country. The Delta variant is now responsible for most of the cases, but cases and deaths are decreasing, and only Rio de Janeiro suffered a new wave due to this variant.

The lack of a new wave of infections across Brazil is clearly a positive development, but we need to understand why this is happening, as by the end of September 2021 only $42 \%$ of the population were fully vaccinated. It may be that the first vaccine dose, combined with prior immunity from natural infection, has helped to contain a third wave in Brazil. Approximately $72 \%$ of the Brazilian population had received at least one dose of the vaccine.

One unexpected development was that the federal government delayed the process of vaccine acquisition in Brazil. When the outbreak caused by the Gamma variant exploded in February 2021, there were vaccines only for healthcare workers and senior citizens. At that point, mainly the CoronaVac (Sinovac) and ChAdOx1 (Oxford-AstraZeneca) vaccines were used, because public vaccine companies in Brazil were able to have contracts to manufacture the vaccines, and they were manufactured in part locally. Unexpected delays in procurement meant that large-scale vaccination of individuals under the age of 60 only started in May 2021, a program that also included the BNT162b2 (BioNTechPfizer) vaccine.

Brazil has a long history of successful vaccine programs and a well-organized system for delivering vaccines across the country, including to remote regions in the Amazon basin. In contrast to some other countries, Brazil does not have a problem with vaccine hesitancy - only with a lack of vaccine. Now that we have the vaccine, immunization has started to move very fast.

One unanswered question concerns the need for boosters. Rollout of a test to measure the population level of immunity can inform the health authorities if and when a vaccine booster will be necessary. Boosters will be impacted by new variants of concern, which can be identified only by continued genomic surveillance. Brazil is contributing to this worldwide sequencing effort, with more than 40,000 sequences submitted to the GISAID database in 2021.

\section{Tim Spector: Symptom tracking}

Tim Spector is a professor of genetic epidemiology at Kings College London, and is lead investigator of the ZOE COVID Study.

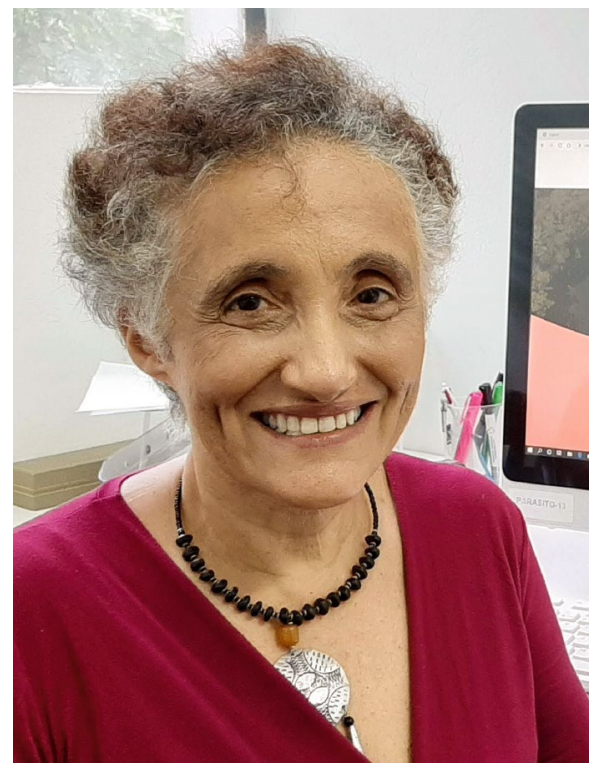

Ester Cerdeira Sabino. Credit: Almir Robson Ferreira, University of São Paulo

In March 2020, we started the ZOE COVID Study to collect information on symptoms from people with suspected COVID-19. This was so that we could predict who had COVID-19 and who didn't, early in the pandemic while testing capacity was low. Four and a half million people downloaded the app in the United Kingdom and United States. One of our biggest discoveries was how loss of smell and taste was predictive of PCR positivity, as well as the risk factors for developing long COVID. With nearly a million people regularly logging in, we've also seen a recent change in symptoms: as the variants and environment change, including weather, seasons and social factors, symptoms are becoming more respiratory and less systemic.

The ZOE team has now identified an incredible range of symptoms, with over 25 symptoms being recognized for the combination of COVID-19 and long COVID. A big question is how the same virus gives some people a skin rash on their toes, but others get gastrointestinal issues, and $40 \%$ get cold-like symptoms, and so on. Why do some symptoms develop three weeks or three months after being infected? SARS-CoV-2 is a mysterious virus, and we need to keep an open mind on why these symptoms are occurring as the science and our knowledge changes.

The marked variations in symptoms and in immune responses, whereby only $80 \%$ of people produce antibodies, could lead to 


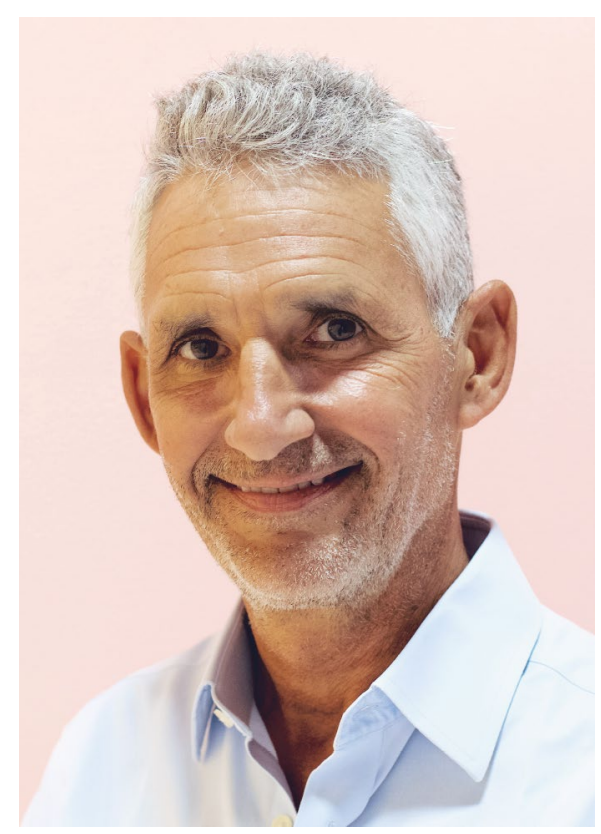

Tim Spector. Credit: Tim Spector

personalized infection advice. We should probably have a personalized risk approach to vaccination, knowing when to get boosters, and selecting the best treatments when someone gets sick. If we start now and use the technology at our disposal, we should be in a much better place for future pandemics.

\section{John Nkengasong: Rebuilding trust}

John Nkengasong is the director of the Africa Centres for Disease Control and Prevention.

The most urgent and important question for COVID-19 in Africa is how to gain access to vaccines. Without a deliberate commitment globally to scale up vaccination in Africa, its people will be threatened by endemic COVID-19 forever. Africa has vaccinated about $5 \%$ of its population in a continent of 1.2 billion people. Africa is dealing with a vaccine famine. Everybody in Africa now knows somebody who has died of COVID-19, so it has become very personal. The solution is producing the vaccines in Africa, but it will take time to build factories and run them appropriately.

Once African nations have access to vaccines, we need to sign a social contract that says 'I will vaccinate myself, because I want to protect myself, and by protecting myself, I protect my loved ones and I protect my community and I protect my country'. Without that, we are very quickly moving toward endemicity, and who wants to live with another endemic disease like AIDS, tuberculosis or malaria?

I'm very encouraged that people across Africa are moving in the right direction, but there are clearly some issues with trust of vaccines. There is a rupture in the relationship between science and the community that to me is very surprising. What we don't know is why anyone might

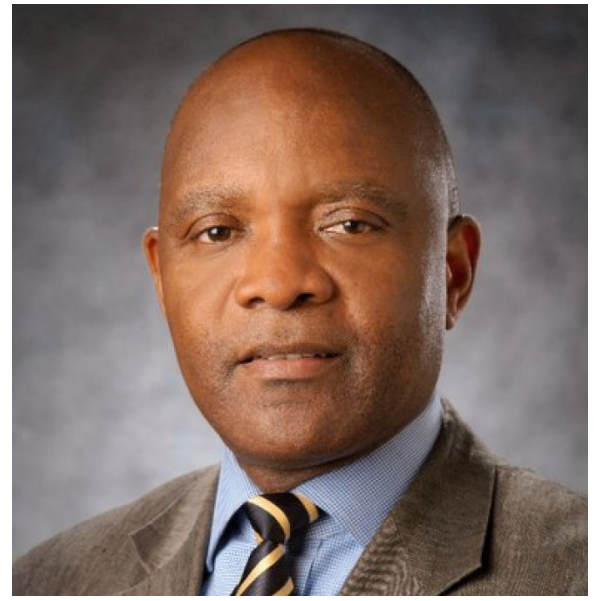

John Nkengasong. Credit: John Nkengasong

not want to get vaccines and how we can have a proper dialogue on how to change that. It should be part of preparing for the next pandemic.

\section{Editorial note}

These interviews have been edited for length and clarity.

Mike May

Freelance writer and editor, Seattle, WA, USA

Published online: 14 December 2021

https://doi.org/10.1038/s41591-021-01598-x 